\title{
Der GPEN Internet Privacy Sweep 2013
}

\section{Datenschutzaufsichtsbehörden überprüfen bei einer internationalen Aktion die Transparenz der Datenverarbeitung durch Websites und Apps}

In der Woche vom 6.-12. Mai 2013 fand der erste weltweite GPEN Internet Privacy Sweep mit dem Thema „Privacy Practice Transperancy“ statt. Bei der Aktion untersuchten die Mitarbeiter von Datenschutzaufsichtsbehörden aus der ganzen Welt, ob die Datenerhebung und -verarbeitung durch Websites und SmartphoneApps für deren Nutzer transparent ist. Organisiert wurde der Sweep vom Global Privacy Enforcement Network (GPEN), einem informellen Zusammenschluss von über 30 internationalen Datenschutzaufsichtsbehörden.

An der Aktion waren insgesamt 19 Datenschutzbehörden aus Europa, Asien, Nordamerika und Ozeanien beteiligt, darunter allein sechs deutsche Aufsichtsbehörden. Auch der Hessische Datenschutzbeauftragte nahm auf Einladung des GPEN am Sweep teil. Während der Sweep-Woche im Mai überprüften die teilnehmenden Behörden weltweit etwa 2000 Websites und SmartphoneApps darauf hin, ob die Angebote über Datenschutzerklärungen verfügen, ob diese leicht auffindbar, verständlich und vollständig sind und ob es einem durchschnittlichen Verbraucher mit üblichem Aufwand möglich ist, sich einen Überblick über die Datenverarbeitung bei den Angeboten zu verschaffen.

Der Hessische Datenschutzbeauftragte hat im Rahmen des Sweep die Datenschutzerklärungen von Websites größerer hessischer Unternehmen überprüft. Das Ergebnis dieser Überprüfung war erfreulicherweise besser als der weltweite Durchschnitt, bei den hessischen Anbietern wurden nur wenige, meist unwesentliche Defizite festgestellt. Kurioserweise fanden sich die schlechtesten Ergebnisse ausgerechnet auf den Websites einer u.a. auf IT-Recht spezialisierten Rechtsanwaltskanzlei und einer Wirtschaftsauskunftei. Der Großteil der vom Hessischen Datenschutzbeauftragten untersuchten Angebote verfügt jedoch über gute bis sehr gute Datenschutzerklärungen, anhand derer die Nutzer ohne großen Aufwand herausfinden können, welche Daten der Anbieter erhebt und wie er diese nutzt. Das gute Abschneiden der hessischen Anbieter ist sicherlich auch auf die vorhandenen gesetzlichen Vorgaben in Deutschland zurückzuführen, nach denen Anbieter von Websites und Apps dazu verpflichtet sind, Informationen zur Datenverarbeitung bereitzustellen. Bei der Untersuchung fiel jedoch auch positiv auf, dass viele
Anbieter Kontaktinformationen von speziell für Datenschutz zuständigen Ansprechpartnern angeben, obwohl es dazu keine gesetzliche Verpflichtung gibt.

Bei der internationalen Auswertung der Ergebnisse des Sweep zeigte sich jedoch, dass etwa ein Fünftel der weltweit überprüften Websites und Apps gar keine Datenschutzerklärung hatten. Nicht wenige Erklärungen enthielten zudem nur allgemeine Informationen oder Textbausteine ohne konkreten Bezug zur Datenerhebung und -verarbeitung auf der jeweiligen Website bzw. bei dem dahinterstehenden Unternehmen.

Noch größere Defizite gab es sogar bei den untersuchten Smartphone-Apps. Mehr als die Hälfte der überprüften Apps hatten gar keine Datenschutzerklärung, die vorhandenen waren fast alle nicht konkret genug oder wiesen andere inhaltliche Mängel auf.

Auch wenn die Prüfung und die Auswertung der Ergebnisse inzwischen abgeschlossen sind, läuft die Nachbereitung des Internet Privacy Sweep 2013 noch weiter. Die teilnehmenden Aufsichtsbehörden ergreifen nunmehr, soweit notwendig, die erforderlichen Schritte, um die festgestellten Defizite zu beseitigen.

Neben der konkreten Überprüfung der Websites und Apps zielte der Sweep unter anderem auch darauf ab, das Bewusstsein der Öffentlichkeit und der Diensteanbieter für den Datenschutz zu schärfen und die internationale Kooperation der Datenschutzaufsichtsbehörden zu fördern. Vor allem rückte die Aktion aber einen Aspekt der Datenverarbeitung in den Fokus, von dem Menschen auf der ganzen Welt als Internet- und Smartphonenutzer täglich betroffen sind. Besonders was die Nutzung von oftmals internationalen Angeboten wie Websites und Apps angeht, erfordert die effektive Durchsetzung des Datenschutzes in einer vernetzten Welt auch international koordiniertes Handeln. Auch in Zukunft soll es deshalb weitere weltweite Aktionen des GPEN geben. So ist für nächstes Jahr bereits ein weiterer Sweep zu einem anderen Aspekt des Datenschutzes geplant.

Martin Buchter

Referent beim Hessischen Datenschutzbeauftragten 